\title{
One species, two developmental modes: a case of geographic poecilogony in marine gastropods
}

\author{
Benedikt Wiggering ${ }^{*}$ (D), Marco T. Neiber®D, Katharina Gebauer and Matthias Glaubrecht
}

\begin{abstract}
Background: Poecilogony, the presence of two developmental modes in the same animal species, is a rare phenomenon. Few cases of poecilogony have been suggested for marine invertebrates including molluscs and even less stood extensive testing, mostly revealing a species pair with differing developmental modes. We studied a textbook example of poecilogony in the viviparous snail Planaxis sulcatus (Gastropoda: Planaxidae), for the first time throughout its entire distribution range.

Results: In the Western Indian Ocean and Red Sea this intertidal species is observed to have large, shelled juveniles, whereas in the Indo-West Pacific planktotrophic veliger larvae are released from a subhaemocoelic brood pouch. We uncovered a shift in developmental modes across its range: from west to east successively earlier developmental stages are released. Furthermore, genetic data based on mitochondrial DNA suggests to recognize $P$. sulcatus as a single species rather than a group of cryptic species. A reconstruction of the ancestral area of $P$. sulcatus based on molecular data outlines the Western Indian Ocean and the Indo-West Pacific as area of origin.

Conclusion: The findings supporting Planaxis sulcatus as a single widespread species and the geographical shift from one reproductive mode to another suggest for this species to truly represent a case of geographic poecilogony, i.e. differing developmental modes between populations of the same species. Furthermore, the results of our ancestral range estimation imply the release of planktotrophic larvae as the ancestral developmental mode.
\end{abstract}

Keywords: Reproductive biology, Poecilogony, Cryptic species, Larval development, Viviparity, Planaxidae, Gastropoda

\section{Background}

Developmental biology and diversity on a geographic scale are major fields of evolutionary biology studies as they oftentimes influence the interpretation and recognition of groups of individuals as species [1]. Thereby, cases where a polymorphic development is present, are of great interest as they influence our view on species recognition and interpretation of biological phenomena. Poecilogony, the presence of two different developmental modes with

\footnotetext{
* Correspondence: benedikt.wiggering@uni-hamburg.de Department of Animal Diversity, Center of Natural History (CeNak), Universität Hamburg, Martin-Luther-King-Platz 3, 20146 Hamburg, Germany
}

alternative larval types [2-8], is one of these phenomena. Such differing larval types are e.g. planktotrophic larvae (free floating larvae that feed on plankton) or lecithotrophic larvae (larvae that are nursed exclusively by the yolk originally contained within the egg). Some interpretations of the definition of poecilogony require phenotypic differences between larval types and the possibility to encounter singular female specimens producing both types of larvae in the same brood [5, 6]. Others (including the very first), differentiate geographic poecilogony including cases with phenotypically indifferent larvae but differing developmental modes while such differences are found among rather than within populations $[2-4,7,8]$.

(c) The Author(s). 2020 Open Access This article is licensed under a Creative Commons Attribution 4.0 International License, which permits use, sharing, adaptation, distribution and reproduction in any medium or format, as long as you give appropriate credit to the original author(s) and the source, provide a link to the Creative Commons licence, and indicate if changes were made. The images or other third party material in this article are included in the article's Creative Commons licence, unless indicated otherwise in a credit line to the material. If material is not included in the article's Creative Commons licence and your intended use is not permitted by statutory regulation or exceeds the permitted use, you will need to obtain permission directly from the copyright holder. To view a copy of this licence, visit http://creativecommons.org/licenses/by/4.0/. The Creative Commons Public Domain Dedication waiver (http://creativecommons.org/publicdomain/zero/1.0/) applies to the data made available in this article, unless otherwise stated in a credit line to the data. 
Only thirteen cases of poecilogony in marine organisms have been thoroughly studied and supported (seven spionid polychaetes $[9,10]$, five sacoglossan gastropods [11] and one littorinimorph gastropod [12]). For molluscs, a total of 42 cases have been suggested [5, 6], though only six cases are supported [11, 12]. The remaining cases of proposed poecilogony are either rejected or in need of detailed investigation.

The marine snail Planaxis sulcatus (Born, 1780) is a textbook example for poecilogony [13]. This viviparous intertidal species is widespread throughout the tropical Indo-West Pacific Region (IWP, geographic delimitations following [14]), with populations known from the Red Sea (RS), Western Indian Ocean (WIO) and IndoPolynesian (IP) provinces. In this species, fertilized eggs are kept in a subhaemocoelic brood pouch (i.e. located in the neck region of the headfoot) until larvae or juveniles are released. Based on records form the Iranian Gulf and Pakistan populations, it has been suggested that $P$. sulcatus releasing juveniles are adelphophagic (offspring grow large by consuming other earlier developmental stages or other juveniles within the mother's brood pouch), supplying nutrition eggs for juveniles within the brood pouch [15-17]. Concordantly, in these populations it was found that broods do not represent single cohorts, but rather that eggs can be added sequentially [17]. As in these populations later developmental stages (viz. juveniles) are far larger than earlier stages (viz. larvae), an adelphophagic nourishment is highly probable. However, no differences in larval morphology between veliger and juvenile releasing specimens has been recorded [15-20].

Six reports on the reproductive biology of this species have been published - all from singular, geographically scattered populations. In the Persian Gulf [15] and in Pakistan [16, 17] populations with long breeding periods releasing comparably large, shelled juveniles were found, whereas in Japan, Thailand [18], New Caledonia [19] and Northeast Australia [20] only planktotrophic veliger larvae were reported. These different developmental modes were either considered as an indication for poecilogony $[13,15,21]$, or as revealing the presence of cryptic species [6]. In all populations studied, reproductive modes were the same within populations, differing only among populations. However, a thorough investigation of the reproductive modes throughout this species' whole range is currently lacking, as is a molecular genetic study on potentially present cryptic species.

In this study we aim at evaluating the developmental modes in P. sulcatus, for the first time encompassing specimens from 71 populations across its entire range, in context with analysing molecular genetic data to clarify the potential existence of cryptic species. To investigate the geographic origin of this species and its plesiomorphic developmental mode, we conducted an ancestral range estimation based on the molecular data.

\section{Results \\ Reproductive biology}

Developmental modes of $P$. sulcatus differ distinctly between geographic regions (Fig. 1). Brood pouches of IP populations contained large amounts of larvae (offspring only exhibiting a protoconch). By contrast, in RS and WIO populations, both larval stages and large juveniles (shelled offspring with a teleoconch) were present. Of the overall 364 dissected specimens 64 (17,6\%) were gravid females (for a detailed list of each studied specimen and their exact brood pouch content refer to Table S3).

The number of offspring found in each brood pouch varied broadly, ranging from as few as 28 up to 10,500 individuals. Irrespective of the geographic origin, larval stages were always found in large amounts, whereas fewer individuals were present in each brood pouch when juveniles in an advanced state of development were present. In general, we found that the further developed the ontogenetic stages within a brood pouch, the fewer the amount of offspring therein.

The largest size class (juveniles $1-1.5 \mathrm{~mm}$ ) was exclusively found within the RS populations. Regularly, all offspring within each individual brood pouch were of the same developmental stage and size class. Only $12.6 \%$ of all gravid females harboured more than one size class of offspring. In these cases, a mixture of successive size classes (for instance an admixture of $0.6-1 \mathrm{~mm}$ juveniles and $1.1-1.5 \mathrm{~mm}$ juveniles) was found.

Only within one Yemenite and one Eritrean population (both RS) one gravid female each was present containing a mixture of larval and juvenile stages within their brood pouches. The Yemenite specimen (CWR 129/84-4) contained a mixture of late larvae $(n=15)$ and small juveniles $(<0.5 \mathrm{~mm}, n=80)$ within its brood pouch. By contrast, the Eritrean specimen (SMF 346144-4) contained a mixture of few late larvae $(n=$ $35)$ and a relatively many large juveniles $(0.6-1.5 \mathrm{~mm}$, $n=80$ ) within its brood pouch. Other than these two specimens, we never encountered females from any populations containing both larvae and juveniles within the same brood pouch. Furthermore, we did not find any phenotypic differences between larvae from WIO and IP populations with different reproductive modes.

\section{Molecular genetics}

Our genetic study yielded $2816 \mathrm{~S}$ and 16 COI sequences from a total of $32 \mathrm{P}$. sulcatus specimens (see Table S2 for a list of these specimens and GenBank accession numbers of individual sequences). Therefore, $19.6 \%$ of the 163 genetically sampled specimens yielded genetic data. The 32 specimens that were successfully amplified 


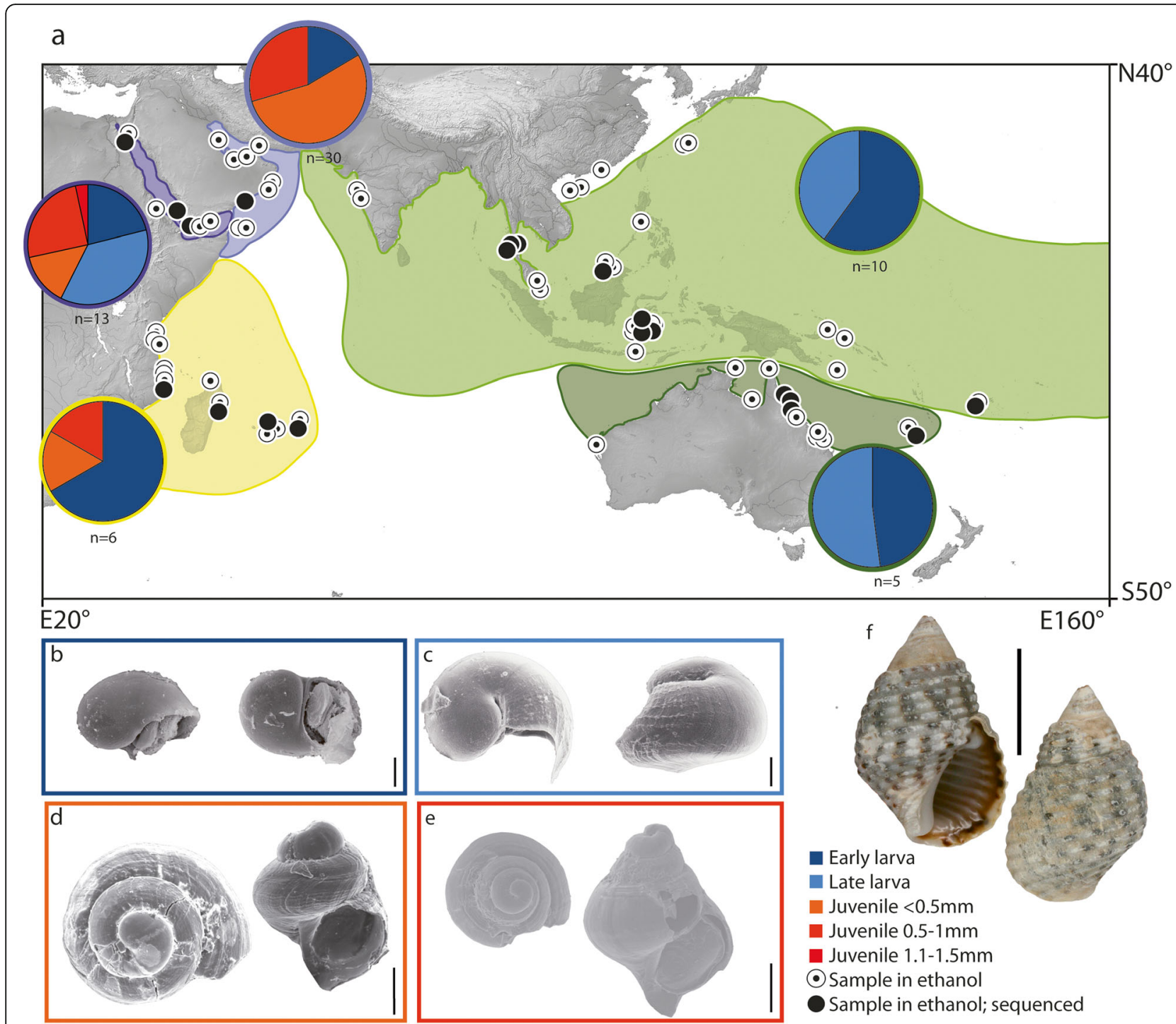

Fig. 1 Distribution area of Planaxis sulcatus, (a) with brood pouch content per geographic area and (b-f) (SEM-) pictures of ontogenetic stages of Planaxis sulcatus. a. Pie charts show the cumulative proportion of each developmental stage size class in brood pouches within the five biogeographic areas, indicated by coloured shades. Numbers below each diagram indicates the number of gravid females within each region. Black dots indicate genetic samples. b-e SEM-pictures of ontogenetic stages of Planaxis sulcatus (Born, 1780) arranged according to size classes, in apertual and top view. b. Early larva, Malaysia, Borneo (ZMB 108275); c. Late larva, Tanzania (ZMB 108265); d. Juvenile < 0.5 mm, Oman, Sud (ZMB 107849); e. Juvenile 0.5-1 mm, Oman, Masirah (ZMB 107850); f. Adult, Indonesia, Southeast Sulawesi (ZMB 106003-13). Scale bars: b, c. = $30 \mu \mathrm{m} ; \mathbf{d} .=100 \mu \mathrm{m} ; \mathbf{e} .=200 \mu \mathrm{m} ; \mathbf{f} .=1 \mathrm{~cm}$. Map made with Natural Earth. Free vector and raster map data @ naturalearthdata.com; for details refer to methods section

and sequenced originated from 20 populations, with eleven populations represented by one, seven populations represented by two and two populations represented by three specimens. The molecular phylogeny based on all available sequences of $P$. sulcatus revealed three distinct clades within this taxon (Fig. 2): one consisting of IP specimens (clade I), being sister to the other two, a second clade including specimens from the RS and northern WIO (clade II) and its sister clade consisting of specimens from southern WIO and IP (clade III).
The ABGD approach revealed that up to three groups can be distinguished within our COI dataset. At a K2Pvalue of $0.1 \%$ three groups were distinguished, aligning with clades I-III, at $1.3 \%$ two groups were found, one representing clade I and another representing clades II and III. At 3.08\% all partitions collapsed (see Table S4 for more details).

The bGMYC approach based on the COI dataset suggested the same groupings as the ABGD approach (see Fig. S1 for a probability matrix based on the BEAST COI tree). 


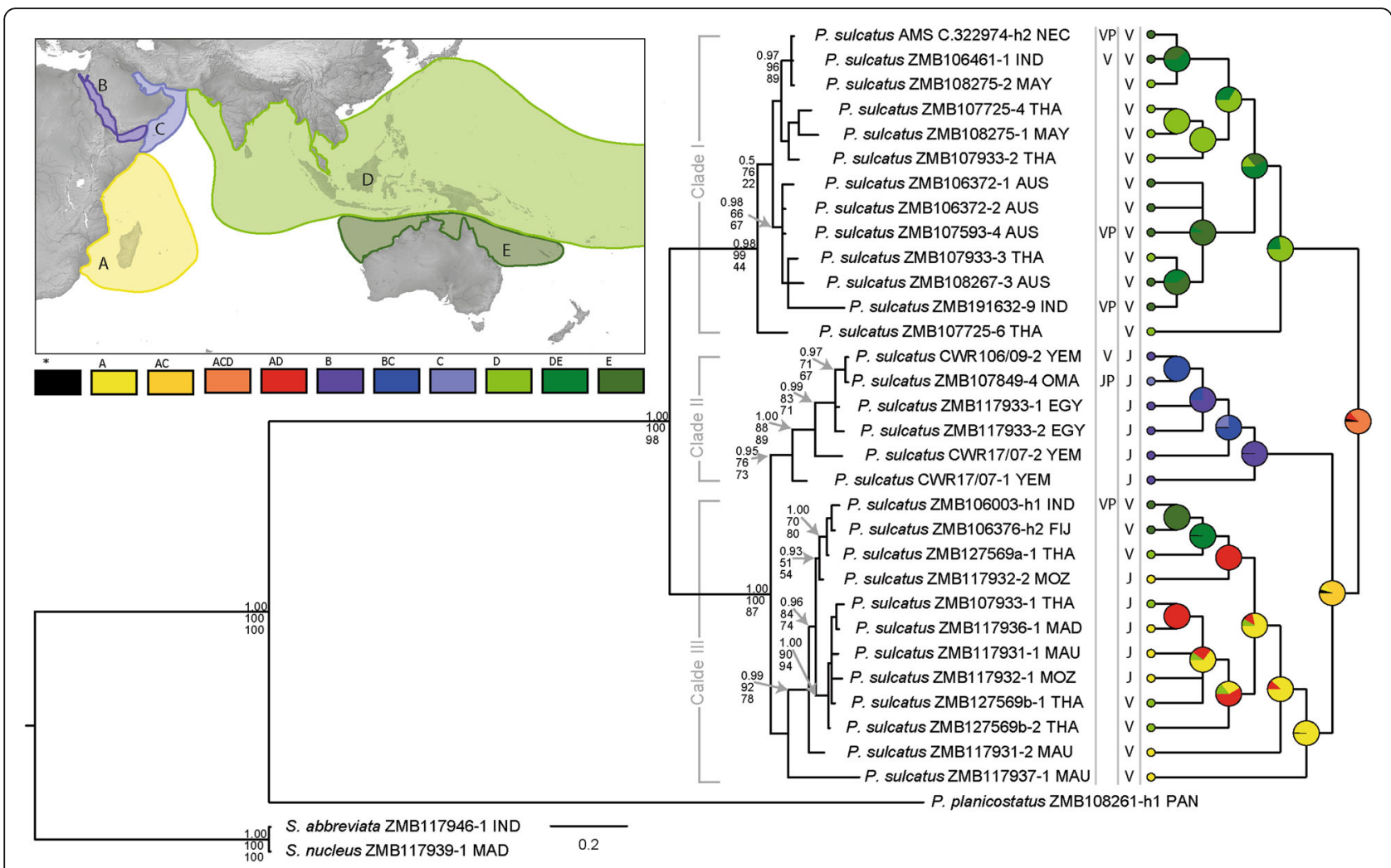

Fig. 2 Bayesian 50\% majority-rule consensus tree based on concatenated COI and 165 sequence data (left topology); nodes displaying the posterior probability (PP) values (top) from the Bayesian inference (BI), bootstrap support (BS) values from the maximum parsimony (MP) analysis (middle) and from the maximum likelihood (ML) analysis (bottom). Only nodes with PP values $\geq 0.95$ and/or BS values from ML and/or MP analyses $\geq 70 \%$ are annotated. Columns to the right of the mitochondrial tree represent reproductive mode found in an individual or within the population of the sequenced individual (left column) and assumed reproductive mode based on area of origin (right column). Empty lines indicate absence of gravid females in the population. Abbreviations: $V=$ veliger stages, $V P=$ population found with veliger stages, $J=$ juveniles, $J P=$ population found with juveniles. Right topology: ancestral range estimation, with pie charts at nodes representing the estimated proportions for each area of origin. Colour codes correspond to areas depicted in the map: A= Southern West Indian Ocean, B=Red Sea, C = Northern West Indian Ocean, $\mathrm{D}=$ Northern Indo-West Pacific, $\mathrm{E}=$ Australia and New Caledonia. A combination of letters represents a shared probability of ancestral range from the corresponding regions. Abbreviations for countries of sample origin: AUS = Australia, EGY $=E g y p t, F I J=F i j i, I N D=$ Indonesia, $\mathrm{YEM}=$ Yemen, $\mathrm{MAU}=$ Mauritius, $\mathrm{MAD}=$ Madagascar, $\mathrm{MAY}=$ Malaysia, MOZ = Mozambique, NEC = New Caledonia, OMA = Oman, $\mathrm{PAN}=$ Panama, THA = Thailand. Map made with Natural Earth. Free vector and raster map data @ naturalearthdata.com; for details refer to methods section

The ancestral range estimation suggests a highly probable combined IP and WIO origin (RASP Value (SV) = 86.68\%) of P. sulcatus (Fig. 2). The different genetic clades, however, show different probabilities of origin: clade I is of mainly IP ( $\mathrm{SV}=77.35 \%)$ origin, whereas clade II is proposed to be of mainly Northern WIO origin $(\mathrm{SV}=99.58 \%)$. Lastly, although consisting of samples from Southern IP and Southern WIO specimens, clade II is highly probable to be of combined Southern WIO and RS (SV $=86.23 \%)$ origin.

\section{Discussion}

This study corroborates the presence of two developmental modes within P. sulcatus: one with large amounts of planktotrophic veliger larvae being released, and a second one with small amounts of shelled juveniles hatching from the female's brood pouch. Contrasting prior studies that provided snapshots of distant populations [15-20], our more comprehensive study uncovered a gradually changing pattern of variation within developmental modes across the entire distribution range (Fig. 1). Generally, the further to the east and south the smaller were the juvenile shells within brood pouches with only larval stages being present in populations east of Pakistan (i.e. IP populations). Within the RS only juvenile stages were present. However, in the WIO both, juveniles and larvae, were found.

A co-occurrence of larval and juvenile stages within the same brood pouch was only observed at one Yemenite and one Eritrean locality. The Yeminite population contained one gravid female with late larvae and small juveniles (CWR 129/84-4). In this case we assume that 
all larvae of this specimen would have developed into juveniles, rather than being released as a mixture of veligers and juveniles as the encountered offspring was from consecutive developmental stages and class sizes. In other populations in this region, much larger juveniles were present, lending support for our assumption. The Eritrean specimen (SMF 346144-4) is an outstanding exception, as the presence of early larvae and large juveniles $(0.6-1.5 \mathrm{~mm})$ could potentially be interpreted as two reproductive modes being present. As specimens from both this Yemenite and the Eritrean population did not yield any molecular data, we cannot provide further insights into these critical populations. Neither our nor any other study on $P$. sulcatus indicated in any other case the presence of both reproductive modes within the same population [15-20].

Interpreting larval stages within brood pouches of conserved specimens as direct implications for present reproductive mode could, however, be ambiguous. As each specimen is sampled at a fixed point in time, there is no saying if larvae would have been released as veliger or developed further into juveniles within the brood pouch. However, the alternative - determining reproductive mode by observing the hatching/release of offspring from brood pouches directly - is logistically very challenging, due to the geographic scope of the present study. As our samples have been collected between 1921 and 2013, and throughout different seasons of these years, we regard the potential hypotheses that brood pouch content patterns are just coincidental as unlikely and, accordingly, assume the approach to infer reproductive mode by this trait as valid.

The findings of the Eritrean specimen (SMF 3461444) containing both early larvae and large juveniles could alternatively be a hint at adelphophagy in $P$. sulcatus, as it has been suggested that larger offspring feed on developing eggs (so called nurse eggs) [15-17]. This is further hinted at by the observation that the further developed the ontogenetic stages within a brood pouch are the fewer the amount of offspring present therein. This correlation might be an indication to not only nurse eggs being consumed by developing offspring but rather all kinds of siblings might be the nourishment of the offspring. In contrast to other studies [15-17], we did not find any distinct nurse eggs. Therefore, to unambiguously solve the question of offspring nourishment in $P$. sulcatus further studies, expanding the limits of our dataset, are needed.

The genetic study reveals three clades of $P$. sulcatus (Fig. 2). The ABGD approach showed that the maximum $\mathrm{K} 2 \mathrm{P}$-distance within the COI gene of all specimens is $3.08 \%$. Other marine caenogastropods show an intraspecific variance between 0.31 and $4.11 \%$. For instance, the intraspecific variance of two similarly distributed littorinid species is 1.36 and $3.48 \%$ [22]. The bGMYC approach suggested similar groupings as the ABGD approach (Fig. S1). It must be noted that molecular species delimitation models work best for groups with a low intraspecific and high interspecific variation. In other cases, these methods usually show a tendency to oversplitting [23]. The potential intraspecific variance of $3.08 \%$ that we found for $P$. sulcatus might suggest that population structure is detected in this case rather than distinct species. Except for developmental modes, no morphological differentiation within P. sulcatus can be observed [see also 15, 17, 18, 20] and a geographic restriction without partial overlap is not present within the molecular clades. Furthermore, developmental modes do not entirely correlate with genetic clades. Hence, we see the genetic differences found in our study to represent intraspecific variation and reject the hypothesis [6] that $P$. sulcatus should be considered as a complex of cryptic species.

However, only $36 \%$ of the individuals, from which DNA was extracted proved to be suitable for PCR amplification. Besides the protocol listed in the material and method section $[24,25]$ we tried other protocols that, however, all failed to extract any additional specimens. As our study was based on museum material sampled between 1921 and 2013, with most samples collected during the 1980s and 1990s, a major hurdle of this study was finding primers and extraction protocols that actually yield DNA given this rather "old" - i.e. degraded museum material. Consequently, an exact genetic coverage of each gravid specimen could not be realized. Hence, we interpret genetic and brood pouch content information as proxies for their respective geographic areas, rather than as direct information obtained from specific individuals in all cases.

Alternatively, the two or three genetic lineages in $P$. sulcatus could be interpreted as representing distinct species. However, this still would include at least one lineage in which the two differing developmental modes are present (see clade III in Fig. 2). Following the notion of not unnecessarily multiplying additional arguments (and taxa), we here refrain from expanding our discussion beyond the scope covered by our data, which hint at the existence of only one poecilogonous species. Planaxis sulcatus therefore exhibits a developmental mode, in which planktotrophic veliger larvae are released (predominant in IP populations) and another where larvae are retained for a longer period only to be released as juveniles (predominant in WIO and RS populations).

We did not find any obvious morphological differences between larval stages, excluding poecilogony in P. sulcatus according to the strict definition suggested by Hoagland \& Robertson [5]. However, following the original definition of poecilogony [3] and more recent discussions [8], we adhere to a broader definition of the term where no morphological 
difference but only a polymorphism of developmental modes is the necessary criterion. Consequently, we see $P$. sulcatus to represent a case of geographic poecilogony.

Finally, the results of the ancestral range estimations imply a shared geographic origin of Planaxis sulcatus from IP and WIO (Fig. 2). As most other Planaxidae release veliger larvae from their brood pouches, it was assumed that this is the plesiomorphic mode of reproduction in this family [20]. Therefore, it appears likely that the spawning of pelagic veliger larvae is the ancestral developmental mode in P. sulcatus as well, as this is the main developmental mode of $P$. sulcatus in the ancestral regions.

Thus, we infer a scenario where from the centre of the IP adjacent areas have been colonised by ancestral lineages of this species. These consecutively evolved the developmental mode we now see within WIO and RS populations. Brooding larger juveniles may have ensured a higher rate of survival by sheltering the progeny from environmental conditions being different in the WIO and RS.

Prior studies suggested higher sea surface salinity [6] and intertidal zoning [17] as factors influencing the emergence of different reproductive modes. Especially changing salinities might be important as it has often been proposed that the evolution of viviparity in freshwater snails is correlated with adaptation to lower salinities [21]. However, in P. sulcatus it would be an increase in salinity triggering the same response. Although these factors are plausible, sea current activity might play an important role as well, as veligers are more prone to sea current drift. In a nonpoeciolognous species it has been shown that depending on substrate (as an indicator for sea current activity), different life history stages hatch from laid eggs [26]. A similar factor might be at hand here, where sea currents are unfavourable for dispersal of WIO and RS populations and, hence, the longer breeding in this area evolved. In other species, poecilogony was proposed as a bet-hedging strategy, to evade unstable conditions in mudflat estuaries [27]. As P. sulcatus inhabits intertidal rocky shores, a similar effect might be possible.

However, to further identify the ecological drivers behind geographic poecilogony in P. sulcatus, controlled aquaria experiments should be conducted. Life samples from WIO, RS and IP populations could each be held under the same salinity and temperature conditions, examining at which stage each population releases its offspring, potentially revealing one of the factors as a trigger for one of the developmental modes. Unfortunately, our current dataset is not suitable to address this question. However, we anticipate that poecilogony in $P$. sulcatus is a direct result of an ecological component leading to and maintaining these differential modes of development.

\section{Conclusion}

Our study implies the presence of poecilogony in the widespread Planaxis sulcatus, with a pattern of geographic variation from one developmental mode to another across its distribution range, varying in the developmental stage at which offspring are released. The ancestral range estimation shows that $P$. sulcatus originated in the WIO or IP, thereby suggesting the release of larval stages to be the initial reproductive mode. Hence, instead of assuming cryptic species our study corroborates $P$. sulcatus to truly represent a textbook example of poecilogony.

\section{Methods \\ Material}

We studied museum specimens of $P$. sulcatus, originating from a total of 71 populations from throughout the IWP, accumulated from several independent collecting trips of MG during the 1990th to early 2000 years, now stored in the Museum für Naturkunde, Berlin (ZMB, Germany), supplemented by material from several other museum collections, including ZMB, Universität Rostock (CWR, Germany), Senckenberg Naturmuseum, Frankfurt (SMF, Germany), Australian Museum, Sydney (AMS) and the Natural History Museum, London (NHMUK, United Kingdom). These samples were collected between 1921 and 2013, though most samples (42) were collected before the year 2000 (see Table S1 for a detailed list of all used samples and sample locations).

\section{(a) Analysis of brood pouch content for developmental mode evaluation}

We studied brood pouch contents of gravid females, to identify reproductive modes within $P$. sulcatus populations. As all previous studies on the matter [15-20] never identified a co-occurrence of reproductive modes within the same population, we interpret finding only larval stages within brood pouches of a given population to represent the veliger releasing mode, whereas we regard populations containing any juvenile stages within brood pouches as indication of direct development. However, this interpretation is not unambiguous as the brood pouch contents only provide a snapshot of the developmental stages present within a population at the time of sampling. Larval stages could still develop into juveniles within the brood pouch or be released at an earlier stage. To account for this ambiguity we combined results from singular populations with other populations from the same area, to infer the reproductive biology in a given region. As our samples were collected between 1921 and 2013 it is highly unlikely that for any given region all samples were collected during the same season, reducing the possibility of a seasonal sampling bias. 
In total, 365 adult specimens were dissected using a Leica M125 stereo microscope (Leica Microsystems $\mathrm{GmbH}$, Wetzlar, Germany). If present, ontogenetic stages were extracted and counted according to predefined size classes: early larva, late larva, juveniles $<0.5$ $\mathrm{mm}$, juveniles $0.5-1 \mathrm{~mm}$, juveniles $1-1.5 \mathrm{~mm}$; see Fig. 2 b-e. We here define larvae as individuals with only a protoconch and juveniles as individuals with a teleoconch. This transition is easily spotted in P. sulcatus as the transition from teleoconch to protoconch is marked by a deep sinusigeral notch [20]. Following Nation [28], larval and juvenile stages were dried for subsequent imaging with a LEO 1525 GEMINI scanning electron microscope (SEM, LEO Electron Microscopy Inc., Thornwood, NY, USA). Dried larval and juvenile stages were mounted on SEM object stubs (Agar Scientific Ltd., Stansted, UK) and coated with platinum using a Polaron SC7640 sputter coater (Quorum Technologies Ltd., Ashford, UK).

\section{DNA extraction \& amplification}

DNA was extracted from foot muscle tissue using mollusc specific protocols [24, 25]. Wherever possible specimens with offspring of known developmental mode in their brood pouches were preferentially used. We performed extractions on 2-3 specimens per studied population, amounting to a total 163 genetically sampled specimens. We added sequences of a confamilial species to our dataset as outgroup: Supplanaxis abbreviata (Pease, 1865) (see Table S1 for a comprehensive list of used samples). Partial sequences of the mitochondrial cytochrome $\mathrm{c}$ oxidase subunit I (COI) gene, with primers LCO1490 (5'-GGT CAA CAA ATC ATA AAG ATA TTG G-3' [29]) and HCO2198var (5'-TAW ACT TCT GGG TGK CCA AAR AAA T-3' [30]), and 16S rRNA (16S) gene, with primers 16SF (5'-CCG CAC TAG TGA TAG CTA GTT TC-3' [31]) and H3059var (5'-CCG GTY TGA ACT CAG ATC ATG T-3' [31]) were amplified by polymerase chain reaction (PCR). Amplifications were performed in $20 \mu \mathrm{l}$ volumes containing $2 \mu \mathrm{l}$ 10x DreamTaq Green Buffer, $0.1 \mu \mathrm{l}$ DreamTaq DNA polymerase (both Thermo Fisher Scientific, Waltham, MA, USA), 0.4 $\mu$ l dNTP mix $10 \mathrm{mM}$ each (VWR chemicals, VWR International GmbH, Darmstadt, Germany), $1 \mu \mathrm{l}$ of each primer (Sigma-Aldrich Chemie GmbH,

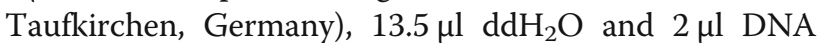
template under the following reaction conditions: initial denaturation at $94{ }^{\circ} \mathrm{C}$ for $3 \mathrm{~min}, 35$ PCR cycles $\left(94^{\circ} \mathrm{C}\right.$ for $30 \mathrm{~s}, 50^{\circ} \mathrm{C}$ for $45 \mathrm{~s}, 72^{\circ} \mathrm{C}$ for $\left.1 \mathrm{~min}\right)$, final extension at $72{ }^{\circ} \mathrm{C}$ for $10 \mathrm{~min}$. PCR products $(10 \mu \mathrm{l})$ were cleaned enzymatically by adding $2 \mu \mathrm{l}$ FastAP Thermosensitive Alkaline Phosphatase $(1 \mathrm{U} / \mu \mathrm{l})$ and $1 \mu \mathrm{l}$ Exonuclease I (20 U/ $\mu l)$ (both Thermo Fisher Scientific) followed by an incubation step at $37^{\circ} \mathrm{C}$ for $15 \mathrm{~min}$ and inactivation at $85^{\circ} \mathrm{C}$ for $15 \mathrm{~min}$. All amplified products were sequenced at Macrogen Europe (Amsterdam, The Netherlands). See Table S2 for GenBank accession numbers for all obtained sequences.

\section{Phylogenetic analysis}

DNA sequences were edited and assembled using GENEIOUS R 9.1.3 (Biomatters Ltd., Auckland, New Zealand). Primer sequences were removed resulting in $\mathrm{COI}$ sequences of $\sim 658 \mathrm{bp}$ and $16 \mathrm{~S}$ sequences of $\sim 810 \mathrm{bp}$. COI sequences were aligned using MUSCLE [32] as implemented in GENEIOUS under default settings. For the $16 \mathrm{~S}$ alignment MAFFT 7 [33], using the Q-INS-I algorithm, the $1 \mathrm{PAM} / \mathrm{K}=2$ option for the scoring matrix for nucleotide sequences and otherwise default settings, was used. We used PartitionFinder 2.1.1 [34] to select the appropriate partitions and evolutionary models. Four partitions were assumed initially (1st, 2nd and 3rd codon positions of COI and 16S). The results of the PartitionFinder analysis using the Bayesian information criterion suggested a single partition and the HKY + G model, which was used for the subsequent Bayesian inference (BI) and maximum likelihood (ML).

We performed a BI using MrBayes version 3.2.6 [35] running Metropolis-coupled Monte Carlo Markov chain $\left(\mathrm{MC}^{3}\right)$ searches with four chains in two separate runs for $50,000,000$ generations with trees sampled every 1000 generations under default heating. Potential scale reduction factors close to 1 and estimated effective sample sizes above 200 from the MrBayes output were used as diagnostics to ensure that the $\mathrm{MC}^{3}$ searches had reached stationarity and convergence. The first 5,000,000 generations of each run were discarded as burn-in. We performed heuristic ML analyses in GARLI 2.0 [36] using the best-fit model as suggested by PartitionFinder. Support values were computed by bootstrapping with 1000 replications. Using PAUP* 4.0b10 [37], we conducted heuristic maximum parsimony (MP) searches with unordered characters, 100 random sequence addition replicates, the tree bisection and re-connection (TBR) branch-swapping, and gaps treated as missing data. Internal branch support was assessed in PAUP* by bootstrapping with 1000 replications, using full heuristic searches with 10 random addition sequence replicates, TBR branch swapping, and one tree held at each step during stepwise addition. Posterior probabilities from the BI analysis and bootstrap support (BS) values from the ML and MP analyses were mapped onto the BI 50\% majority-rule consensus tree with SumTrees version 3.3.1 (part of the DendroPy 3.8.0 package [38]). BS $\geq 70 \%$ from the ML and MP analyses and posterior probabilities $(\mathrm{PP}) \geq 0.95$ were interpreted as positive support for a node. 


\section{Molecular species delimitation models}

To test for the presence of potential cryptic lineages we used the Automated Barcode Gap Discovery (ABGD method) [39], via its online application (http://wwwabi. snv.jussieu.fr/public/abgd/abgdweb.html). For the COI gene we used the Kimura (K80) TS/TV 2.0 distance (K2P). We entered previously established [22] minimum $(\mathrm{Pmin}=0.0031)$ and maximum $(\mathrm{Pmax}=0.0411) \mathrm{COI}$ $\mathrm{K} 2 \mathrm{P}$ values for marine caenogastropod snails (Littorinidae) and otherwise default settings.

We also used the general mixed Yule-coalescent (GMYC) approach in its Bayesian implementation (bGMYC) [40] for DNA sequence-based species delimitation. We constructed ultrametric trees based on COI data with BEAST 2.4.1 [41]. The chain was run for $11,000,000$ generations, with a sample frequency of 1000. The first 1000,000 of the generations were discarded as burn-in. The GTR + I + G model was applied; otherwise default settings were used. Tracer 1.7.1 [42] was used to check that all effective sample sizes were above 200. GMYC and bGMYC analyses were conducted with the Split [43] and bGMYC R packages [44], respectively. The single-threshold as well as the multiplethreshold analyses were both conducted using the maximum clade credibility tree from the BEAST analysis constructed with TreeAnnotator 2.1.2 (part of the BEAST software suite) setting the posterior probability limit to 0 . The bGMYC analysis was based on 100 trees drawn equidistantly from the post burn-in generations obtained from the BEAST analysis. For each of the 100 trees, the Markov-chain Monte Carlo sampler was run for 100,000 generations, discarding the first 90,000 generations as burn-in and sampling every 100 generations.

\section{Ancestral range estimation}

An ancestral range estimation was conducted based on 1000 randomly selected post-burn-in trees from the BI analysis of the dataset accounting for statistical uncertainty and the 50\% majority-rule consensus tree from the $\mathrm{BI}$ analysis using the statistical dispersal-vicariance analysis (S-Diva) method [45] implemented in RASP 4.0 beta [46]. We constructed a matrix in which each individual was assigned to one of five geographic regions (roughly following [14]): A) Southwest Africa, Madagascar and Mauritius; B) the Red Sea; C) the Arabian Sea (including the Gulf of Yemen); D) the IndoWest Pacific (including Indonesia, Malaysia and Thailand) and; E) Australia and New Caledonia. For our analysis, we allowed transitions from B only to A and C, as well as from $\mathrm{E}$ only to $\mathrm{D}$. All other areas were assumed as directly connected. The analysis was run allowing a maximum of three areas at each node and otherwise default settings.

\section{Distribution maps and figure assembly}

The species' distribution was reconstructed on a dot by dot basis based on ethanol stored specimens from the aforementioned collections, with maps based on the open access Natural Earth map (free vector and raster map data@ naturalearthdata.com). Figures and maps were assembled using Adobe Photoshop CS2 version 9.0 for Windows and Adobe Illustrator CS2 version 12.0 for Windows (both Adobe Systems, San Jose, CA, USA).

\section{Supplementary information}

Supplementary information accompanies this paper at https://doi.org/10. 1186/s12862-020-01644-1.

Additional file 1 Table S1 Material examined. Table S2 Specimens examined for the molecular genetic analysis of this study with GenBank accession and inventory numbers. Table S3 Results of brood pouch content analysis of Planaxis sulcatus. If present, content was counted according to five predefined size classes. Table S4 Results of ABGD analysis for the COI mt rRNA dataset using the K2P model to calculate pairwise distances. $P$ is the prior maximum intraspecific divergence. Fig. S1 Pobability matrix of the bGMYC analysis based on the COI mt rRNA dataset. Colours correspond to $p$-values depicted at the right side. Clades are entered corresponding to those shown in Fig. 2.

\section{Abbreviations \\ 16S: $16 S$ rRNA gene; ABGD: Automated Barcode Gap Discovery; AMS: Australian Museum, Sydney; AUS: Australia; bGMYC: Baysian general mixed Yule-coalescent approach; BI: Bayesian inference; BS: Bootstrap support; COI: Mitochondrial cytochrome c oxidase subunit I gene; CWR: Universität Rostock (Germany); DNA: Deoxyribonucleic acid; EGY: Egypt; FIJ: Fiji; GMYC: General mixed Yule-coalescent approach; IND: Indonesia; IP: Indo-Polynesian Province; J: Juveniles; JP: Population found with juveniles; K2P: Kimura (K80) TS/TV 2.0 distance; MAU: Mauritius; MAD: Madagascar; MAY: Malaysia; MC $^{3}$ : Metropolis-coupled Monte Carlo Markov chain; ML: Maximum likelihood; MOZ: Mozambique; MP: Maximum parsimony; NEC: New Caledonia; NHMUK: Natural History Museum, London (United Kingdom); OMA: Oman; PAN: Panama; PCR: Polymerase chain reaction; rRNA: Ribosomal ribonucleic acid; RS: Red Sea Province; S-Diva: Statistical dispersal-vicariance analysis; SEM: Scanning electron microscope; SMF: Senckenberg Naturmuseum, Frankfurt (Germany); SV: RASP Value; TBR: Tree bisection and re-connection branch-swapping; THA: Thailand; V: Veliger stages; VP: Population found with veliger stages; WIO: Western Indian Ocean Province; YEM: Yemen; ZMB: Museum für Naturkunde, Berlin (Germany)}

\section{Acknowledgements}

We thank Ronald Janssen (Senckenberg Naturmuseum, Frankfurt), Frank Köhler (Australian Museum, Sydney), Andreia Salvador (Natural History Museum, London), Christian Wranik (Universität Rostock) and Christine Zorn (Museum für Naturkunde, Berlin) for providing museum samples. We thank two anonymous reviewers for their constructive criticism on earlier versions of the manuscript, as well as Stephane Guindon for very helpful editorial support.

\section{Authors' contributions}

MG designed the study, organized funding, collected ZMB material and coauthored paper drafts. BW carried out morphological studies and wrote the manuscript. KG carried out the molecular lab work. MN and BW analysed the data. BW, MG, MN and KG proof read manuscript drafts and final versions. All authors have read and approved the manuscript for submission and publication.

Funding

No specific funding was involved. 


\section{Availability of data and materials}

The morphological datasets supporting the conclusions of this article are included within the article and its additional files.

The genetic dataset supporting the conclusions of this article is available in the GenBank repository, see Table S2 of the supporting files for accession numbers.

\section{Ethics approval and consent to participate}

Our study is based exclusively on museum material collected in and mainly before the year 2013. We have a permission to collect the samples from the museum (listed in the methods section) and adhered to the specified terms of use of the respective collections.

\section{Consent for publication}

Not applicable.

\section{Competing interests}

We declare to have no competing interests.

\section{Received: 4 February 2020 Accepted: 18 June 2020}

Published online: 26 June 2020

\section{References}

1. Minelli A. Species diversity vs. morphological disparity in the light of evolutionary developmental biology: table 1. Ann Bot. 2016;117:781-94.

2. Giard MA. Sur le bourgeonnement des laves d'Astellium spongiforme Gd. et sur la poecilogonie chez les ascidies composées. C R Acad Sci. 1891;112(1): 301-4.

3. Giard MA. Nouvelies remarques sur la poecilogonie. C R Hebd Seanc Acad Sci Paris. 1892:114:1549-52.

4. Giard MA. La poecilogony. In: Bedot M, editor. Compte-Rendu des Séances du Sixième Congrès International de Zoologie. Geneva: Imprimerie W. Kündig \& Fils; 1905. p. 617-46.

5. Hoagland $K E$, Robertson R. An assessment of poecilogony in marine invertebrates: phenomenon or fantasy? Biol Bull. 1988;174(2):109-25.

6. Bouchet P. A review of poecilogony in gastropods. J Molluscan Stud. 1989; 55:67-78.

7. Chia FS, Gibson G, Qian PY. Poecilogony as a reproductive strategy of marine invertebrates. Oceanol Acta. 1996;19(3-4):203-8.

8. Knott KE, McHugh D. Introduction to symposium: Poecilogony—a window on larval evolutionary transitions in marine invertebrates. Integr Comp Biol. 2012;52(1):120-7.

9. Collin R. Nontraditional life-history choices: what can "intermediates" tell us about evolutionary transitions between modes of invertebrate development? Integr Comp Biol. 2012;52(1):128-37.

10. Oyarzun FX, Brante A. A new case of poecilogony from South America and the implications of nurse eggs, capsule structure, and maternal brooding behavior on the development of different larval types. Biol Bull. 2015;228(2):85-97.

11. Vendetti JE, Trowbridge CD, Krug PJ. Poecilogony and population genetic structure in Elysia pusilla (Heterobranchia: Sacoglossa), and reproductive data for five sacoglossans that express dimorphisms in larval development. Integr Comp Biol. 2012;52(1):138-50.

12. McDonald KA, Collin R, Lesoway MP. Poecilogony in the caenogastropod Calyptraea lichen (Mollusca: Gastropoda). Invertebr Biol. 2014;133(3):213-20.

13. Tardent P. 5. Fortpflanzungsbiologie 5.3.2. Invertebrata. In: Meeresbiologie Eine Einführung. Stuttgart: Thieme; 1979. p. 295-305.

14. Bowen BW, Gaither MR, DiBattista JD, lacchei M, Andrews KR, Grant WS, et al. Comparative phylogeography of the ocean planet. PNAS. 2016;113(29): 7962-9.

15. Thorson G. Studies on egg masses and larval development of Gastropoda from the Iranian Gulf. In: Jessen K, Sporck R, editors. Danish Scientific Investigations in Iran Part II. Copenhagen; 1940. p. 159-238.

16. Barkati S, Ahmed M. Studies on the reproductive biology of some prosobranchs from the coast of Karachi (Parkistan) bordering the northern Arabian Sea. I. Observations on Planaxis sulcatus (Born, 1780). Veliger. 1982; 24(4):355-8.

17. Ahmed M, Siddiqui G. Histology of gonads and cephalic brood pouch of the marine gastropod Planaxis sulcatus of Karachi coast (Pakistan). Hydrobiologia. 1997;350:127-30.

18. Ohgaki S-I. Some aspects of the breeding biology of Planaxis sulcatus (Born) (Gastropoda: Planaxidae). J Molluscan Stud. 1997;63(1):49-56.
19. Risbec J. Biologie et ponte de mollusques gastéropodes Néo-Calédoniens. Bull Soc Zool Fr. 1936;60:388-417.

20. Houbrick RS. Anatomy, reproductive biology, and phylogeny of the Planaxidae (Cerithiacea: Prosobranchia). Smithson Contrib Zool. 1987;445:1-57.

21. Glaubrecht M. Evolutionsökologie und Systematik am Beispiel von Süß- und Brackwasserschnecken (Mollusca: Caenogastropoda: Cerithioidea): Ontogenese-Strategien, paläontologische Befunde und Historische Zoogeographie. Leiden: Backhuys Publishers; 1996.

22. Reid DG, Dyal P, Williams ST. Global diversification of mangrove fauna: a molecular phylogeny of Littoraria (Gastropoda: Littorinidae). Mol Phylogenet Evol. 2010;55(1):185-201.

23. Pentinsaari M, Vos R, Mutanen M. Algorithmic single-locus species delimitation: effects of sampling effort, variation and nonmonophyly in four methods and 1870 species of beetles. Mol Ecol Resour. 2017;17:393-404.

24. Sokolov EP. An improved method for DNA isolation from mucopolysacchariderich molluscan tissues. J Molluscan Stud. 2000;66:573-5.

25. Scheel BM, Hausdorf B. Survival and differentiation of subspecies of the land snail Charpentieria itala in mountain refuges in the southern Alps. Mol Ecol. 2012;21(15):3794-808

26. Allen RM. Oviposition site influences dispersal potential in a marine bubble snail. Mar Biol Res. 2014:10:515-22.

27. Krug PJ. Not my "type": larval dispersal dimorphisms and bet-hedging in opisthobranch life histories. Biol Bull. 2009;216:355-72.

28. Nation JL. A new method using hexamethyldisilazane for preparation of soft insect tissues for scanning electron microscopy. Stain Technol. 1983;58(6):347-51.

29. Folmer O, Black M, Lutz R, Vrijenhoek R. DNA primers for amplification of mitochondrial cytochrome c oxidase subunit I from diverse metazoan invertebrates. Mol Mar Biol Biotechnol. 1994;3(5):294-9.

30. von Rintelen T, Wilson AB, Meyer A, Glaubrecht M. Escalation and trophic specialization drive adaptive radiation of freshwater gastropods in ancient lakes on Sulawesi, Indonesia. Proc R Soc Lond B Biol Sci. 2004;271 (1557):2541-9.

31. Wilson $A B$, Glaubrecht $M$, Meyer $A$. Ancient lakes as evolutionary reservoirs: evidence from the thalassoid gastropods of Lake Tanganyika. Proc R Soc BBiol Sci. 2004;271(1538):529-36

32. Edgar RC. MUSCLE: a multiple sequence alignment method with reduced time and space complexity. BMC Bioinform. 2004;5:113.

33. Katoh K, Standley DM. MAFFT multiple sequence alignment software version 7: improvements in performance and usability. Mol Biol Evol. 2013; 30(4):772-80.

34. Lanfear R, Calcott B, Ho SYW, Guindon S. PartitionFinder: combined selection of partitioning schemes and substitution models for phylogenetic analyses. Mol Biol Evol. 2012;29(6):1695-701.

35. Ronquist F, Teslenko M, van der Mark P, Ayres DL, Darling A, Höhna S, et al. MrBayes 3.2: efficient Bayesian phylogenetic inference and model choice across a large model space. Syst Biol. Mai 2012;61(3):539-42.

36. Zwickl DJ. Genetic algorithm approaches for the phylogenetic analysis of large biological sequence datasets under the maximum likelihood criterion: University of Texas Austin; 2006. https://repositories.lib.utexas.edu/bitstream/ handle/2152/2666/zwickld81846.pdf Accessed 3 Dec 2019.

37. Swofford DL. PAUP*: Phylogenetic Analysis Using Parsimony (and other methods) 4.0.b5. Sunderland: Sinauer Associates; 2002. http://citeseerx.ist. psu.edu/viewdoc/summary?doi=10.1.1.458.6867 Accessed 3 Dec 2019.

38. Sukumaran J, Holder MT. DendroPy: a Python library for phylogenetic computing. Bioinformatics. 2010;26(12):1569-71.

39. Puillandre $N$, Lambert $A$, Brouillet $S$, Achaz G. ABGD, automatic barcode gap discovery for primary species delimitation. Mol Ecol. 2012;21(8):1864-77.

40. Reid NM, Carstens BC. Phylogenetic estimation error can decrease the accuracy of species delimitation: a Bayesian implementation of the general mixed Yule-coalescent model. BMC Evol Biol. 2012;12:196.

41. Bouckaert R, Heled J, Kühnert D, Vaughan T, Wu C-H, Xie D, et al. BEAST 2: a software platform for Bayesian evolutionary analysis. PLoS Comput Biol. 2014:10:e1003537.

42. Rambaut A, Drummond AJ, Xie D, Baele G, Suchard MA. Posterior summarization in Bayesian Phylogenetics using Tracer 1.7. Syst Biol. 2018;67: 901-4.

43. Ezard THG, Thomas GH, Purvis A. Inclusion of a near-complete fossil record reveals speciation-related molecular evolution. Methods Ecol Evol. 2013;4: $745-53$

44. R Core Team. R: a language and environment for statistical computing: Vienna, R Foundation for Statistical Computing: 2018. Available online at https://www.R-project.org/. 
45. Yu Y, Harris AJ, He X. S-DIVA (statistical dispersal-Vicariance analysis): a tool for inferring biogeographic histories. Mol Phylogenet Evol. 2010;56(2):848-50.

46. Yu Y, Harris AJ, Blair C, He X. RASP (reconstruct ancestral state in

phylogenies): a tool for historical biogeography. Mol Phylogenet Evol. Juni 2015;87:46-9.

\section{Publisher's Note}

Springer Nature remains neutral with regard to jurisdictional claims in published maps and institutional affiliations.

Ready to submit your research? Choose BMC and benefit from:

- fast, convenient online submission

- thorough peer review by experienced researchers in your field

- rapid publication on acceptance

- support for research data, including large and complex data types

- gold Open Access which fosters wider collaboration and increased citations

- maximum visibility for your research: over $100 \mathrm{M}$ website views per year

At $B M C$, research is always in progress.

Learn more biomedcentral.com/submissions 